\title{
Parametrization, characterization, and optimization of double-bend achromat cell
}

\author{
Yunhai Cai \\ SLAC National Accelerator Laboratory, 2575 Sand Hill Road, Menlo Park, California 94205, USA
}

(Received 11 December 2019; accepted 19 February 2020; published 11 March 2020)

\begin{abstract}
We continue the study of single-particle dynamics in a more realistic cell, namely, the double-bend achromat with three families of quadrupoles. The cell is parametrized with the optical parameters including the phase advances and the horizontal beta functions at its entry and center. At zero chromaticity, we find that the landscape of the dynamic aperture in the tune plane can be captured by a simple formula constructed from the effective Hamiltonian of fourth order. Furthermore, the optimal dynamic aperture can be found by simply minimizing the third-order driving terms with equal weight in the effective Hamiltonian.
\end{abstract}

DOI: 10.1103/PhysRevAccelBeams.23.034002

\section{INTRODUCTION}

Four decades ago, modern and dedicated synchrotron light sources based on electron storage rings were initiated by Chasman, Green, and Rowe [1], proposing a double focusing achromat lattice. Essentially, each periodical structure consists of two double-bend achromat (DBA) cells with a wiggler in the middle of the straight. The DBA cell was naturally extended to a triple-bend achromat $[2,3]$. Theoretically, an analysis of the minimum emittance achievable in the achromatic condition was carried out by Sommer [4], who laid a solid foundation for further development of the third-generation light sources [5] that have become the most efficient, versatile, and productive facilities worldwide for the research of materials, life, environmental, and geochemistry sciences.

Despite the tremendous success, the understanding of these lattices is largely limited to the numerical solutions and simulations found by running computer programs such as MAD [6]. The optics design requires hand-on experiences and sometimes good intuition. These experiences, accumulated over the decades, are not adequately documented partially because of a lack of systematic and theoretical approaches. Good progress was made to classify the linear and stable optics with global scanning of the quadrupole settings [7]. The scheme is clearly important for existing facilities but not quite complete for the design of a new lattice, since properly choosing distances between the quadrupoles plays an equally important role in the design of linear optics.

Published by the American Physical Society under the terms of the Creative Commons Attribution 4.0 International license. Further distribution of this work must maintain attribution to the author(s) and the published article's title, journal citation, and DOI.
For nonlinear dynamics, a major advance was the successful introduction of the frequency map analysis [8] to particle accelerators [9]. More importantly, it reveals accurately the relevant resonances, often very high-order ones, in the particle motion. As a result, it becomes a standard tool to characterize [10] the third-generation light sources. One of the main characteristics of the lattice is the dynamic aperture. Often, retaining an adequate acceptance while reducing the emittance is a critical design issue. A breakthrough was made by introducing harmonic sextupoles [11] in the straights to minimize the resonance driving terms [12] and, therefore, to increase the dynamic aperture.

In computation, dedicated codes BETA [13] and OPA [14] were developed to minimize the third- and fourth-order driving terms. These codes have been successfully used to optimize the design of many light sources [15-17]. More recently, utilizing enormous computing power, the multiobjective genetic algorithm [18,19] was introduced and enhanced by machine learning [20] to directly optimize the dynamic aperture. Despite these practical improvements, the relationship between the driving terms and dynamic aperture is still elusive. The optimization remains largely an art. Most of the time, we do not adequately understand the optimal solution found by computers.

In this paper, we will extend our work of the parametrized alternating focusing and defocusing (FODO) [21] and theoretical minimum emittance (TME) [22] cells to a more practical and realistic DBA cell. Again, for simplicity, we will set the chromaticity to zero with two families of sextupoles in the dispersive region. To avoid repetition, our emphasis of nonlinear dynamics will be placed on more practical situations when the betatron tunes are placed sufficiently away from the low-order resonances, and yet these resonances play an important role. Moreover, we will study both analytically and numerically the optimization of the dynamic aperture. Since there is a dispersion-free 
region, we will naturally use harmonic sextupoles for the optimization.

\section{LINEAR OPTICS}

\section{A. Half cell}

We now consider half of a cell with reflection symmetry as shown in Fig. 1, where the position $s_{1}$ is the starting point and $s_{2}$ is the reflecting point. Here we represent the doublet with their geometrical parameters $p, q$, and $f$ in Eq. (1) in Ref. [22], where $w$ is the focus length of the quadrupole at the entry and $H$ and $L$ are the distances between the doublet to $s_{1}$ and $s_{2}$, respectively.

On one hand, using the matrix in Eq. (2) in Ref. [22] for the doublet and the matrices of a drift and thin quadrupole, we compute the transfer matrix from $s_{1}$ to $s_{2}$ and find

$$
M_{S_{1} \rightarrow s_{2}}=\left(\begin{array}{cc}
\frac{(L-q)(H+p-\mathrm{w})-f^{2}}{f \mathrm{w}} & f-\frac{(H+p)(L-q)}{f} \\
\frac{H+p-\mathrm{w}}{f \mathrm{w}} & -\frac{H+p}{f}
\end{array}\right) .
$$

On the other, the matrix can be represented by [23]

$$
M_{s_{1} \rightarrow s_{2}}=\left(\begin{array}{cc}
\sqrt{\frac{\beta_{2}}{\beta_{1}}} \cos \pi \nu & \sqrt{\beta_{1} \beta_{2}} \sin \pi \nu \\
-\frac{1}{\sqrt{\beta_{1} \beta_{2}}} \sin \pi \nu & \sqrt{\frac{\beta_{1}}{\beta_{2}}} \cos \pi \nu
\end{array}\right),
$$

where $\beta_{1}$ and $\beta_{2}$ are the beta functions [24] at the positions $s_{1}$ and $s_{2}$, respectively, and $\nu$ the betatron tune of the cell. Here we have used the property of the reflection points, namely, $\alpha_{1}=\alpha_{2}=0$. Comparing it with the matrix in Eq. (1), we obtain

$$
\begin{aligned}
& p=\frac{(\mathrm{w}-H) \beta_{1} \cos \pi \nu+\mathrm{w} H \sin \pi \nu}{\beta_{1} \cos \pi \nu-\mathrm{w} \sin \pi \nu}, \\
& q=\frac{\left(L \beta_{1}-\mathrm{w} \beta_{2}\right) \cos \pi \nu-\left(\mathrm{w} L+\beta_{1} \beta_{2}\right) \sin \pi \nu}{\beta_{1} \cos \pi \nu-\mathrm{w} \sin \pi \nu}, \\
& f=\frac{\mathrm{w} \sqrt{\beta_{1} \beta_{2}}}{\mathrm{w} \sin \pi \nu-\beta_{1} \cos \pi \nu} .
\end{aligned}
$$

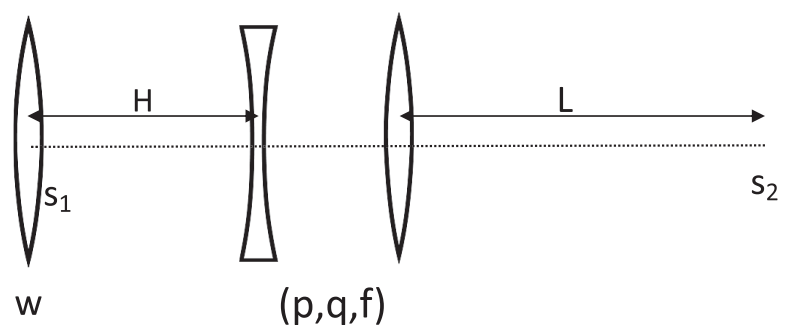

FIG. 1. A schematic layout of half of a cell with reflection symmetry.

The formulas in this section are equally applicable to either the horizontal or vertical plane. In this paper, we choose the horizontal lattice functions to fix physical parameters such as the quadrupole strengths, since one of our main concerns is the natural emittance, which is determined entirely by the parameters of the dipole and the horizontal lattice functions as we will show later.

\section{B. Full cell}

We would like to apply the parametrization of the half cell to a periodic DBA cell that contains five quadrupoles as shown in Fig. 2. $L$ is half of the straight section. The cell is chosen because it contains the most essential ingredients in the common DBA cells and yet is analytically solvable. A focusing quadrupole and a chromatic sextupole are lumped together as a thin multipole at the entry and the exit, while another chromatic sextupole is placed at the end of a sector-bending dipole in the dispersive region. Here $\kappa_{f}$ and $\kappa_{d}$ are integrated strengths of the focusing and defocusing sextupoles, respectively, and $f_{f}$ and $f_{d}$ the focal lengths of the focusing and defocusing quadrupoles, respectively. Also, $\phi$ and $J$ are the bending angle and length of the dipole, respectively.

Given the geometrical parameters in Eq. (3), the focus lengths of the quadrupoles and separation distance of the doublet can be calculated. Substituting Eq. (3) into Eq. (4) in Ref. [22], we obtain

$$
\begin{aligned}
f_{f} & =\frac{\mathrm{w} L \beta_{1}-H L \beta_{1}+\mathrm{w} H \beta_{2}+\left(\mathrm{w} H L+H \beta_{1} \beta_{2}-\mathrm{w} \beta_{1} \beta_{2}\right) \tan \pi \nu}{(\mathrm{w}-H) \beta_{1}-\mathrm{w} \sqrt{\beta_{1} \beta_{2}} \sec \pi \nu+\mathrm{w} H \tan \pi \nu}, \\
f_{d} & =\frac{\left(\mathrm{w} L \beta_{1}-H L \beta_{1}+\mathrm{w} H \beta_{2}\right) \cos \pi \nu+\left(\mathrm{w} H L+H \beta_{1} \beta_{2}-\mathrm{w} \beta_{1} \beta_{2}\right) \sin \pi \nu}{\mathrm{w} \sqrt{\beta_{1} \beta_{2}}+\left(L \beta_{1}-\mathrm{w} \beta_{2}\right) \cos \pi \nu-\left(\mathrm{w} L+\beta_{1} \beta_{2}\right) \sin \pi \nu}, \\
g & =\frac{\left(H L \beta_{1}-\mathrm{w} L \beta_{1}-\mathrm{w} H \beta_{2}\right) \cos \pi \nu-\left(\mathrm{w} H L+H \beta_{1} \beta_{2}-\mathrm{w} \beta_{1} \beta_{2}\right) \sin \pi \nu}{\mathrm{w} \sqrt{\beta_{1} \beta_{2}}},
\end{aligned}
$$

where we have made a substitution of $f_{1}=f_{d}$ and $f_{2}=f_{f}$. 


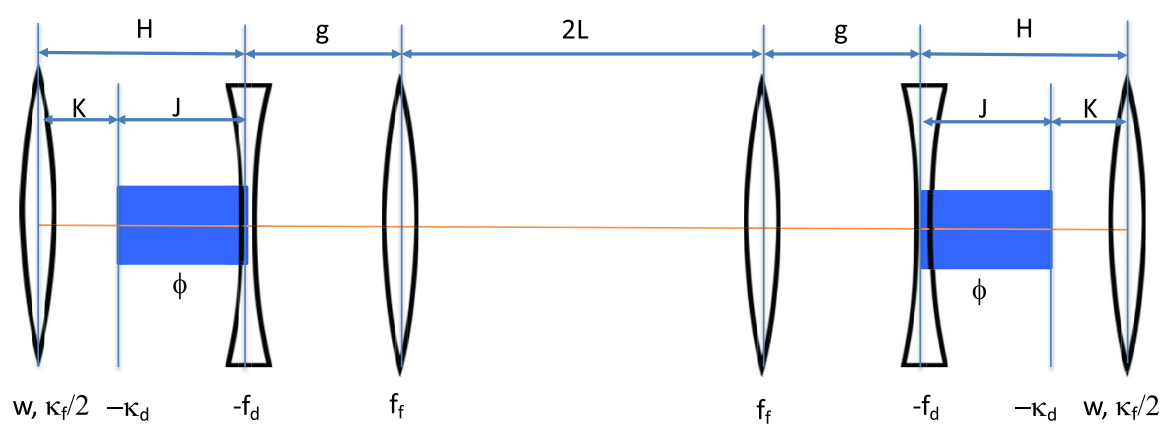

FIG. 2. A periodic double-bend achromat cell with dipole, quadrupole, and sextupole magnets.

\section{Achromat}

Now, we would like to analyze the dispersive region near the end of the DBA cell as shown in Fig. 3. We start at the entry of the bending magnet with zeros for both the horizontal dispersion $\eta_{0}=0$ and its slope $\eta_{0}^{\prime}=0$. Propagating to the exit, using the maps of Eqs. (2.5) and (2.6) in Ref. [25], we have

$$
\eta_{1}=\left(K+\frac{J}{2}\right) \phi, \quad \eta_{1}^{\prime}=-\frac{(J+2 K-2 \mathrm{w}) \phi}{2 \mathrm{w}} .
$$

The periodic condition of the cell requires $\eta_{1}^{\prime}=0$. It can be satisfied by setting the focal length of the quadrupole to

$$
\mathrm{w}=K+\frac{J}{2} .
$$

Basically, the focal length of the quadrupole in the middle of the dispersive region is not a free parameter. It should be equal to the distance to the center of the dipole from the quadrupole.

For the lattice functions, we propagate them backward from the exit with the horizontal beta function $\beta_{1}$ and $\alpha_{1}=0$ and find at the entry of the dipole

$$
\begin{aligned}
& \beta_{0}=\frac{(J+K)^{2}}{\beta_{1}}+\frac{(J+K-\mathrm{w})^{2} \beta_{1}}{\mathrm{w}^{2}}, \\
& \alpha_{0}=-\frac{\beta_{1}}{\mathrm{w}}+(J+K)\left(\frac{1}{\beta_{1}}+\frac{\beta_{1}}{\mathrm{w}^{2}}\right) .
\end{aligned}
$$

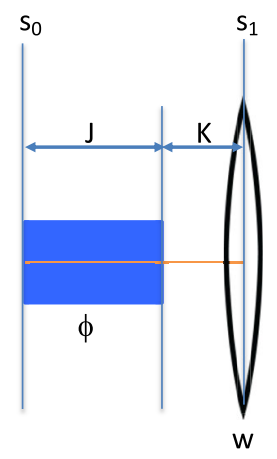

FIG. 3. Half of the dispersive region in the DBA cell.

\section{Emittance}

With $\alpha_{0}$ and $\beta_{0}$, we evaluate the radiation integrals [26] and derive the form factor

$$
F=\frac{8+57 \bar{K}+180 \bar{K}^{3}+80 \bar{K}^{4}+2\left(76 \bar{K}^{2}+\bar{\beta}_{1}^{2}\right)}{60(1+2 \bar{K})^{2} \bar{\beta}_{1}}
$$

which is defined by the natural emittance $\epsilon_{x}=C_{q} F \gamma^{2} \phi^{3}$, where $C_{q}=3.8319 \times 10^{-13} \mathrm{~m}$ and $\gamma$ is the Lorentz factor. Here we have used w in Eq. (6) and defined $\bar{K}=K / J$ and $\bar{\beta}_{1}=\beta_{1} / J$, using a "bar" to note the relative lengths to the dipole length. A contour plot of the form factor is shown in Fig. 4. Clearly, the blue area can be a good choice of the low-emittance lattices.

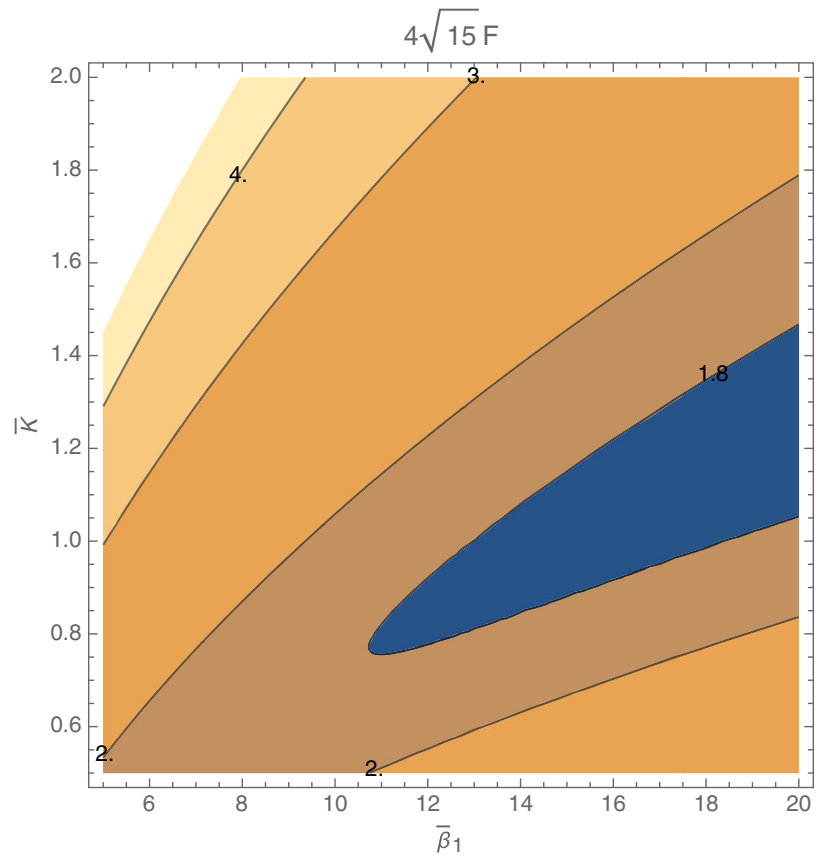

FIG. 4. The form factor of emittance in Eq. (8) as a function of the horizontal beta function at the quadrupole and the distance between the quadrupole and the dipole in the dispersive region. 
Minimizing $F$ with respect to $\bar{\beta}_{1}$, we find the optimum

$$
\bar{\beta}_{1}=\frac{(1+2 \bar{K}) \sqrt{8+25 \bar{K}+20 \bar{K}^{2}}}{\sqrt{2}},
$$

with which the form is reduced to

$$
F=\frac{\sqrt{8+25 \bar{K}+20 \bar{K}^{2}}}{15 \sqrt{2}(1+2 \bar{K})} .
$$

It reaches its minimum $F_{\min }^{(s)}=1 /(3 \sqrt{10})$, as the limit of $\bar{K}$ approaching infinity. Here we use the superscript " $s$ " to indicate that it is valid only for the case of a single quadrupole in the dispersion region. Comparing it to the well-known minimum [4] $F_{\min }=1 /(4 \sqrt{15})$ in the general case, we have

$$
\frac{F_{\min }^{(s)}}{F_{\min }}=2 \sqrt{\frac{2}{3}}
$$

Note that it numerically agrees with the previously known value [27].

\section{E. Stability}

By setting the quadrupoles in the doublet according to the horizontal betatron tune in Eq. (4) and the other quadrupole in the dispersive region with Eq. (6), we ensure stability in the horizontal plane. In the vertical plane, these quadrupoles alter the signs of the focus lengths. Using this property, we find that the vertical tune is given by

$$
\begin{aligned}
\cos 2 \pi \nu_{y}:= & 1-\left\{2 \mathrm{w}^{2} H^{2} L+\mathrm{w}^{3} H L+2 H^{2} L \beta_{1}^{2}-2 \mathrm{w} H L \beta_{1}^{2}+7 \mathrm{w}^{2} H \beta_{1} \beta_{2}\right. \\
& +7 \mathrm{w}^{3} \beta_{1} \beta_{2}-4 \mathrm{w} \sqrt{\beta_{1} \beta_{2}}\left[\beta_{1}\left(\mathrm{w}^{2}-H^{2}-2 H L\right)+\mathrm{w} \beta_{2}(2 H+\mathrm{w})\right] \cos \pi \nu \\
& +\left[\mathrm{w} \beta_{1} \beta_{2}\left(\mathrm{w}^{2}+\mathrm{w} H-4 H^{2}\right)-\mathrm{w}^{2} H L(2 H+\mathrm{w})+2 H L \beta_{1}^{2}(H-\mathrm{w})\right] \cos 2 \pi \nu \\
& -4 \mathrm{w} \sqrt{\beta_{1} \beta_{2}}\left(\mathrm{w} H^{2}+2 \mathrm{w} H L+\mathrm{w}^{2} H+\mathrm{w}^{2} L+2 H \beta_{1} \beta_{2}\right) \sin \pi \nu \\
& \left.+\left[\mathrm{w} L \beta_{1}\left(\mathrm{w} H-4 H^{2}+\mathrm{w}^{2}\right)+\mathrm{w}^{2} H \beta_{2}(2 H+\mathrm{w})+2 H \beta_{1}^{2} \beta_{2}(\mathrm{w}-H)\right] \sin 2 \pi \nu\right\} \\
& \times\left\{6 \mathrm{w} H L^{2} \beta_{1}^{2}-4 \mathrm{w}^{2} H^{2} L^{2}-4 H^{2} L^{2} \beta_{1}^{2}-2 \mathrm{w}^{2} L^{2} \beta_{1}^{2}-7 \mathrm{w}^{2} H L \beta_{1} \beta_{2}\right. \\
& -2 \mathrm{w}^{2} H^{2} \beta_{2}^{2}-2 H^{2} \beta_{1}^{2} \beta_{2}^{2}+3 \mathrm{w} H \beta_{1}^{2} \beta_{2}^{2}-\mathrm{w}^{2} \beta_{1}^{2} \beta_{2}^{2} \\
& +4 \mathrm{w} \sqrt{\beta_{1} \beta_{2}}\left[L \beta_{1}\left(2 \mathrm{w} H+\mathrm{w} L-2 H^{2}-2 H L\right)+\mathrm{w} H \beta_{2}(H+2 L)\right] \cos \pi \nu \\
& +\left[3 \mathrm{w} H L \beta_{1} \beta_{2}(4 H-3 \mathrm{w})+\left(\beta_{2}^{2}-2 L^{2}\right)\left[\beta_{1}^{2}\left(2 H^{2}-3 \mathrm{w} H+\mathrm{w}^{2}\right)-2 \mathrm{w}^{2} H^{2}\right]\right] \cos 2 \pi \nu \\
& +4 \mathrm{w} \sqrt{\beta_{1} \beta_{2}}\left[2 \mathrm{w} H L(H+L)+\beta_{1} \beta_{2}\left(H^{2}+2 H L-\mathrm{w} H-\mathrm{w} L\right)\right] \sin \pi \nu \\
& +\left[2 \mathrm{w} H L\left(4 H L \beta_{1}-3 \mathrm{w} L \beta_{1}-3 \mathrm{w} H \beta_{2}\right)+3 L \beta_{1}^{2} \beta_{2}\left(2 H^{2}-3 \mathrm{w} H+\mathrm{w}^{2}\right)\right. \\
& \left.\left.+\mathrm{w} H \beta_{1} \beta_{2}^{2}(3 \mathrm{w}-4 H)\right] \sin 2 \pi \nu\right\} \\
& /\left\{2 \mathrm{w}^{3} \beta_{1} \beta_{2}\left[\left(L \beta_{1}(\mathrm{w}-H)+\mathrm{w} H \beta_{2}\right) \cos \pi \nu+\left(\mathrm{w} H L+\beta_{1} \beta_{2}(H-\mathrm{w})\right) \sin \pi \nu\right]^{2}\right\} .
\end{aligned}
$$

The calculation can be carried out by either multiplying the matrices or concatenating the maps. This condition has to be satisfied for a stable cell. It defines a cubic equation of $L$, which is the half length of the straight section. The solution is given in the Appendix.

Since $H=J+K$, we have found that seven independent parameters, namely, $\nu_{x}, \nu_{y}, \beta_{1,2}, K, J$, and $\phi$, can fully characterize the stable DBA cell. $\beta_{1}, K$, and $J$ should be selected to minimize the emittance according to Eq. (8) and $\beta_{2}$ to optimize the brightness of the synchrotron light source. Finally, we should have $\phi=\pi / N_{c}$, where $N_{c}$ is the number of cells in the storage ring. Given an energy, $\phi$ should also be consistent with the required emittance.

We check the parametrization for the DBA cell using the setting of the quadrupoles in Eqs. (4) and (6) against the computer program MAD [6]. In particular, we choose the parameters in Table I for an emittance reasonably near the minimum. Here we have the calculated half length of

TABLE I. Independent parameters of the double-bend achromat cell.

\begin{tabular}{lc}
\hline \hline Parameter & Value \\
\hline Dipole length, $J[\mathrm{~m}]$ & 1 \\
Distance from entry to dipole, $K[\mathrm{~m}]$ & 1 \\
Horizontal beta at entry, $\beta_{1}[\mathrm{~m}]$ & 10 \\
Horizontal beta at center, $\beta_{2}[\mathrm{~m}]$ & 1 \\
Bending angle, $\phi$ & $\pi / 22$ \\
Horizontal tune, $\nu_{x}$ & 1.275 \\
Vertical tune, $\nu_{y}$ & 0.31875 \\
\hline \hline
\end{tabular}


the straight $L=3.05918 \mathrm{~m}$ and the form factor $F=$ 0.12537 . These parameters will be used throughout this paper if not mentioned otherwise. With these parameters, we compute the length of the straight and settings of the doublets as a function of the betatron tunes. The results are shown in Fig. 5.
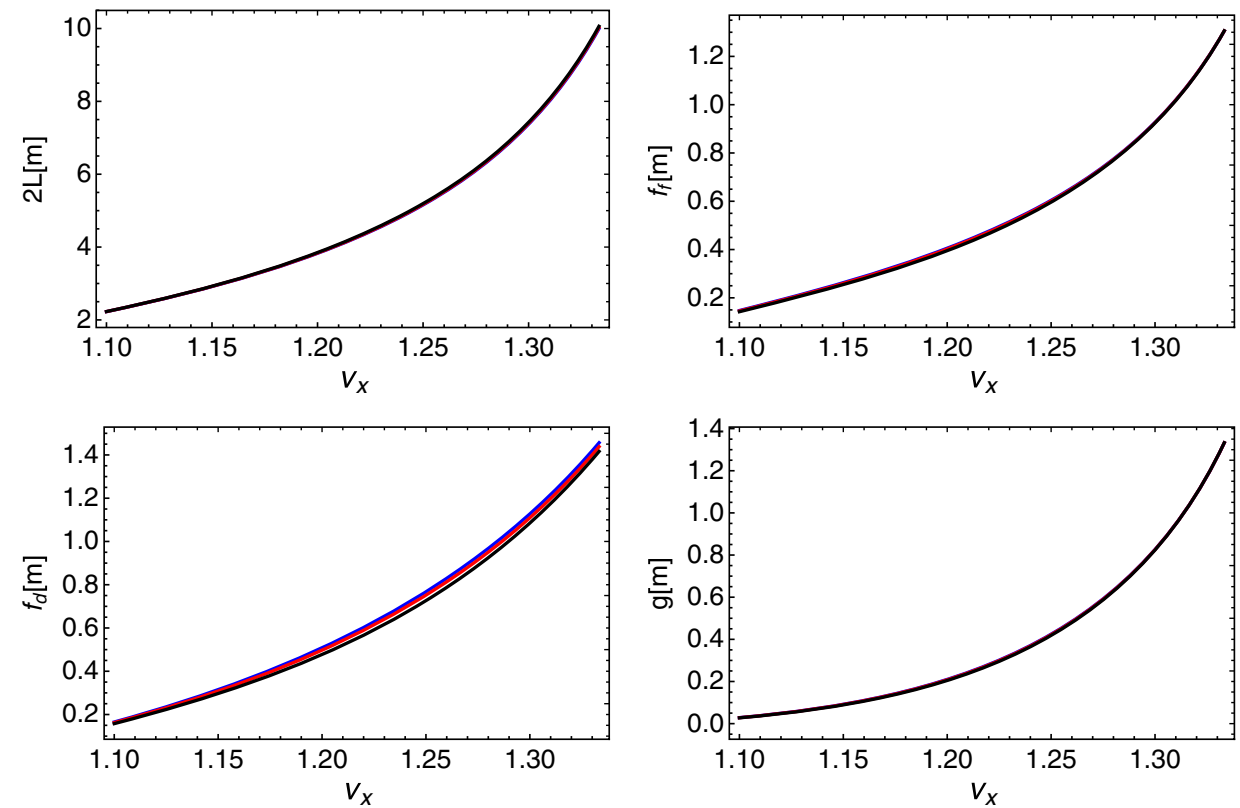

FIG. 5. The length of the straight and the parameters of the doublet as a function of the betatron tunes with various ratios: $\nu_{y}: \nu_{x}=1: 5$, $1: 4$, and $1: 3$ represented by blue, red, and black, respectively.

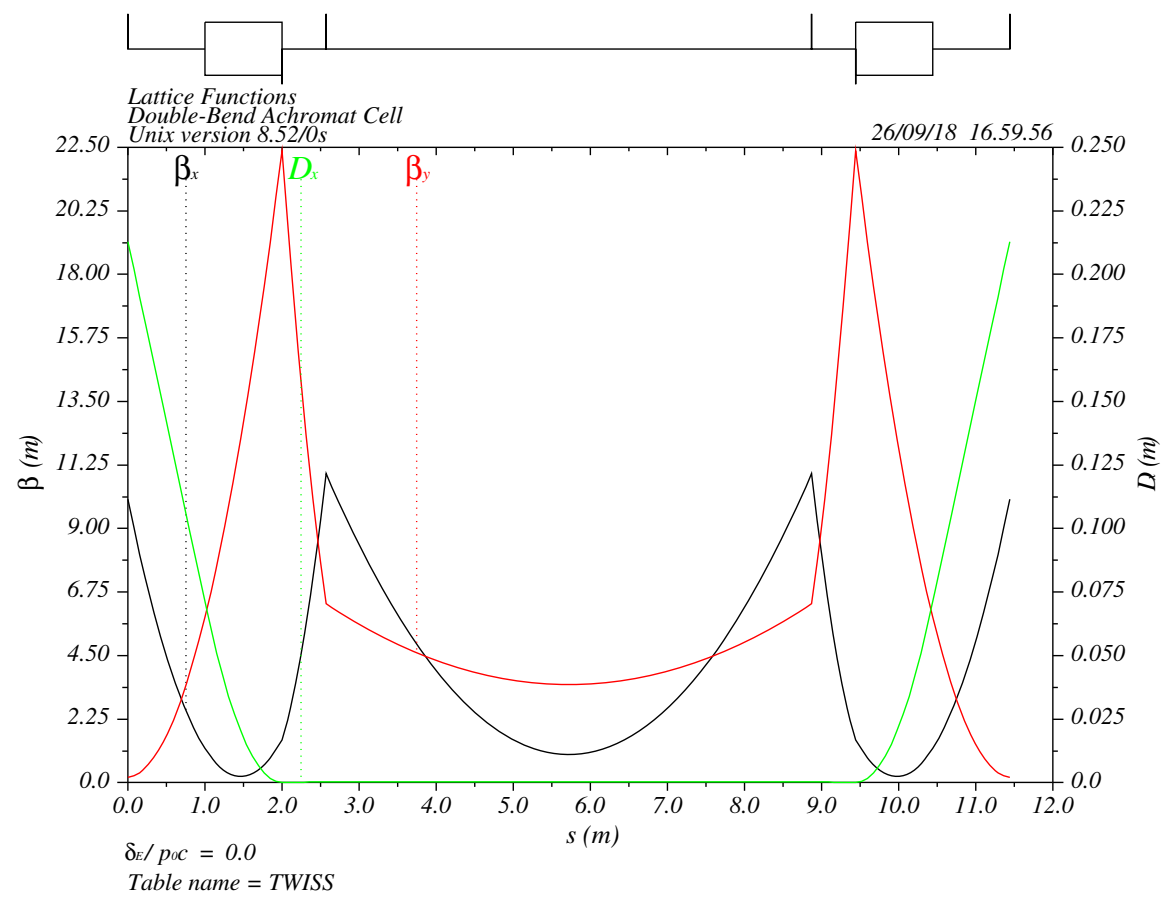

FIG. 6. Lattice functions of the DBA cell with parameters tabulated in Table I. 


\section{NONLINEAR DYNAMICS}

\section{A. Chromatic compensation}

The Courant-Snyder parameters with $\delta$ dependence can be calculated [25] using the symplectic maps. In particular, by computing the phase advances up to the first order of $\delta$, we derive the natural chromaticity. They are plotted in Fig. 7 as a function of the betatron tunes. From the viewpoint of the chromaticity in the vertical plane, the ratio of $\nu_{y}: \nu_{x}=1: 5$ or $1: 4$ seems reasonable, while $1: 3$ is too high, largely due to the high vertical beta function at the defocusing quadrupole.

We can use the two sextupoles to zero out the natural chromaticity. Solving two linear equations, we find the necessary strengths. It is worth noting that the compensation is not local anymore because of the quadrupoles in the straight, where the dispersion is zero. As a result, the formulas of the strengths are very cumbersome. With the formulas, we plot the strengths of the sextupoles in Fig. 8. We can see clearly that the strength largely follows the natural chromaticity in each plane respectively.

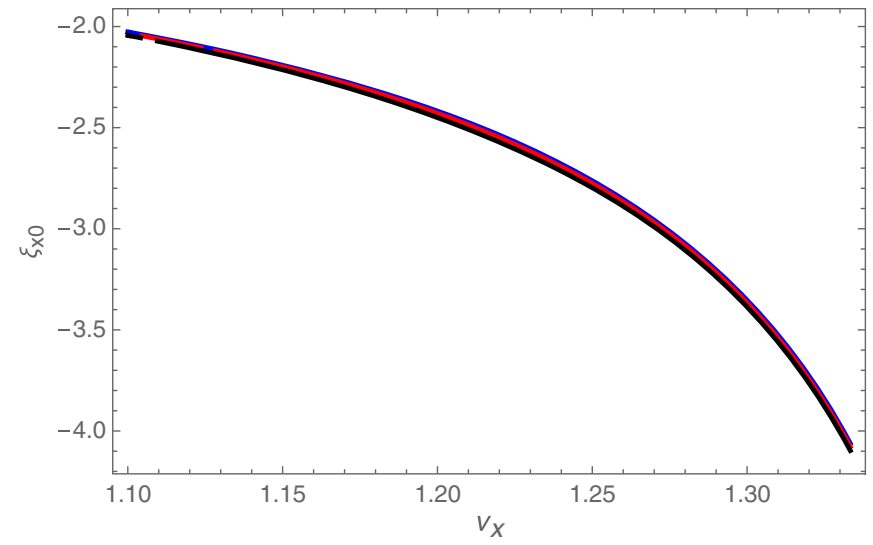

\section{B. Effective Hamiltonian}

Given a symplectic nonlinear map $\mathcal{M}$ in a periodic system, its effective Hamiltonian $\mathcal{H}$ can be defined by [28]

$$
\mathcal{M}=e^{-: \mathcal{H}:}
$$

where we have used the notation of the Lie algebra [29]. The Hamiltonian can be obtained either by using the DragtFinn factorization [30] and the Campbell-Baker-Hausdorff theorem or the normal form [31]. Sometimes, it is also called the pseudo-Hamiltonian. Moreover, it can be written in terms of the polynomials:

$$
\mathcal{H}=\mathcal{H}_{2}+\mathcal{H}_{3}+\mathcal{H}_{4} \cdots
$$

where the subscript indicates the order. At the second order, the Hamiltonian describes the linear harmonic motion:

$$
\mathcal{H}_{2}=2 \pi\left(\Delta \nu_{x} J_{x}+\Delta \nu_{y} J_{y}\right)
$$

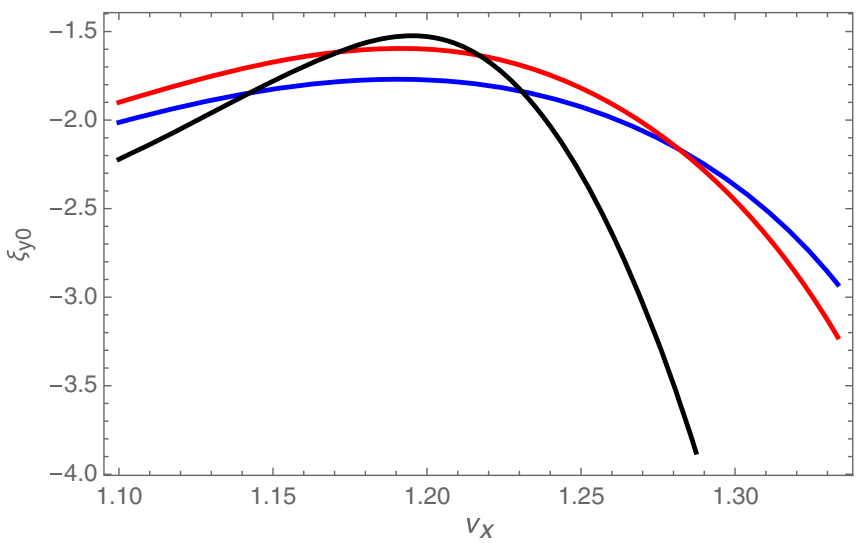

FIG. 7. The natural chromaticity in the horizontal (left) and vertical (right) planes as a function of the betatron tunes with various ratios: $\nu_{y}: \nu_{x}=1: 5,1: 4$, and $1: 3$ represented by blue, red, and black, respectively.
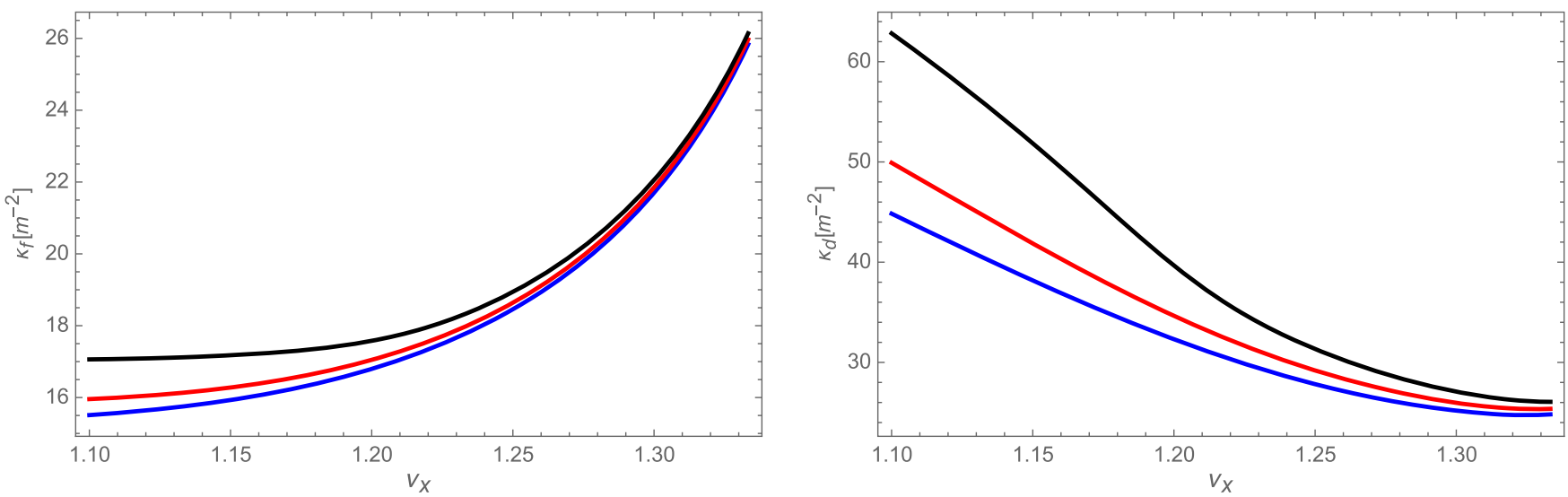

FIG. 8. The settings of focusing (left) and defocusing (right) sextupoles as a function of the betatron tunes with various ratios: $\nu_{y}: \nu_{x}=$ $1: 5,1: 4$, and $1: 3$ represented by blue, red, and black, respectively. 
where $J_{x, y}$ are the action variables [32] and $\Delta \nu_{x, y}$ the fractional part of the betatron tunes in the horizontal and vertical planes, respectively.

The third-order effective Hamiltonian consists of five resonance driving terms and is given by

$$
\begin{aligned}
\mathcal{H}_{3}= & 2 \sqrt{2}\left[C_{3000} J_{x}^{3 / 2} \cos 3 \psi_{x}+C_{2100} J_{x}^{3 / 2} \cos \psi_{x}\right. \\
& +C_{1011} J_{x}^{1 / 2} J_{y} \cos \psi_{x}+C_{1020} J_{x}^{1 / 2} J_{y} \cos \left(\psi_{x}+2 \psi_{y}\right) \\
& \left.+C_{1002} J_{x}^{1 / 2} J_{y} \cos \left(\psi_{x}-2 \psi_{y}\right)\right],
\end{aligned}
$$

where $\psi_{x, y}$ are the angle variables [32]. The coefficients $C_{j k l m}$ are defined to be consistent with the resonance bases [31], and their subscripts indicate the indices of power series in the complex variables. They include small denominators and, therefore, become singular correspondingly at the resonances. Here we have assumed that the reference point has a reflection symmetry; otherwise, there should be corresponding sine terms. For FODO and TME cells, some analytical expressions can be found in Refs. [21,22], respectively.

In the fourth order, the Hamiltonian can be separated into two parts: $\mathcal{H}_{4}=\mathcal{H}_{4}^{(r)}+\mathcal{H}_{4}^{(n)}$. The resonance part is given by

$$
\begin{aligned}
\mathcal{H}_{4}^{(r)}= & 4\left[C_{4000} J_{x}^{2} \cos 4 \psi_{x}+C_{3100} J_{x}^{2} \cos 2 \psi_{x}\right. \\
& +C_{2020} J_{x} J_{y} \cos \left(2 \psi_{x}+2 \psi_{y}\right)+C_{2011} J_{x} J_{y} \cos 2 \psi_{x} \\
& +C_{2002} J_{x} J_{y} \cos \left(2 \psi_{x}-2 \psi_{y}\right)+C_{1120} J_{x} J_{y} \cos 2 \psi_{y} \\
& \left.+C_{0040} J_{y}^{2} \cos 4 \psi_{y}+C_{0031} J_{y}^{2} \cos 2 \psi_{y}\right],
\end{aligned}
$$

and the nonresonance part by

$$
\mathcal{H}_{4}^{(n)}=4\left(C_{2200} J_{x}^{2}+C_{1111} J_{x} J_{y}+C_{0022} J_{y}^{2}\right),
$$

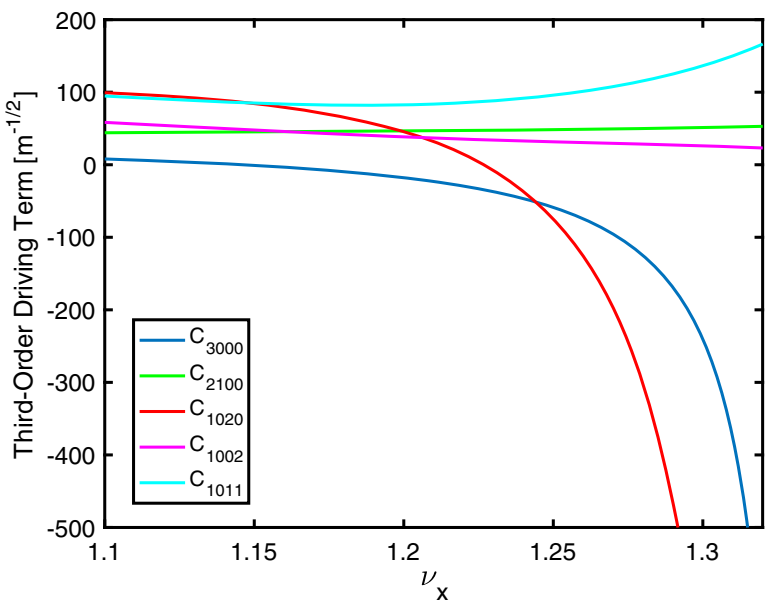

which leads to the detuning for the particles with large betatron amplitudes.

In general, the driving terms can be numerically computed first, using differential algebra [33] to extract the nonlinear map and then the normal form to construct the effective Hamiltonian. In particular, for the DBA cells, they are too complicated to be computed analytically. So we numerically evaluated them and plotted the results in Fig. 9. The singularities of resonances are seen in the plots. For the third order, $3 \nu_{x}$ and $\nu_{x}+2 \nu_{y}$ are clearly the dominant resonances.

\section{Dynamic aperture}

To see the effects of the resonances, we use the formulas in Eqs. (4) and (6) to construct a cell with specified betatron tunes and the parameters in Table I. Setting the sextupoles to make the chromaticity zero, we scan the dynamic aperture by tracking at various betatron tunes. The averaged dynamic aperture in the normalized coordinates is color coded in units of $\mathrm{m}^{1 / 2}$ on the map in the left plot in Fig. 10. The sum resonances $3 \nu_{x}$ and $\nu_{x}+2 \nu_{y}$ are clearly dominant, while the difference resonance $\nu_{x}-2 \nu_{y}$ is barely seen in the tune scan. There is a good region where the dynamic aperture is large. Unfortunately, it cannot be selected, because its straight section is too short to place a undulator as seen in Fig. 5.

In general, the effective Hamiltonian in Eq. (14) is not solvable. Its dynamic apertures of each order can be estimated by

$$
\begin{aligned}
& a_{3}=\frac{\pi \Delta \nu_{x}}{C_{3}^{(r m s)}}, \\
& a_{4}=\sqrt{\frac{(\sqrt{2}-1) \pi \Delta \nu_{x}}{2 C_{4}^{(r m s)}}},
\end{aligned}
$$

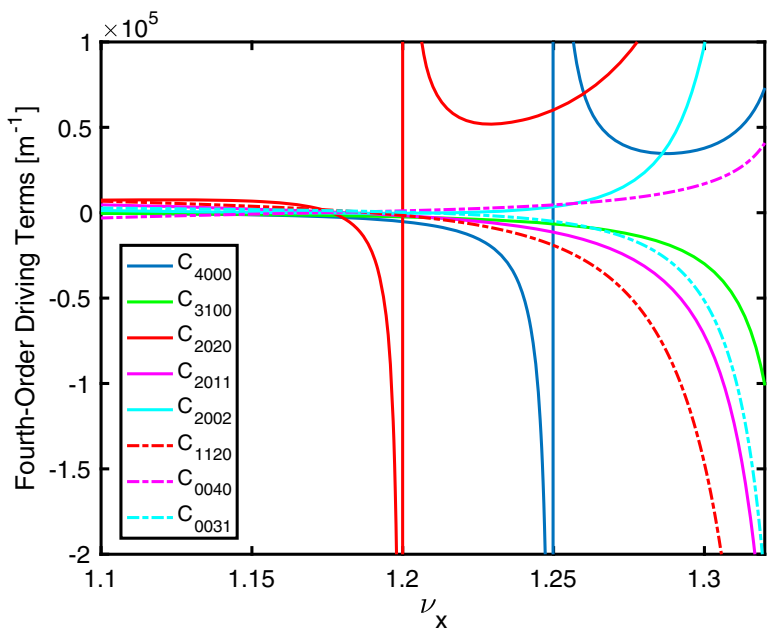

FIG. 9. The driving terms of the third- (left) and fourth-order (right) resonances as a function of the horizontal betatron tune $\nu_{x}$ with a fixed ratio to the vertical tune $\nu_{y}=\nu_{x} / 4$. 

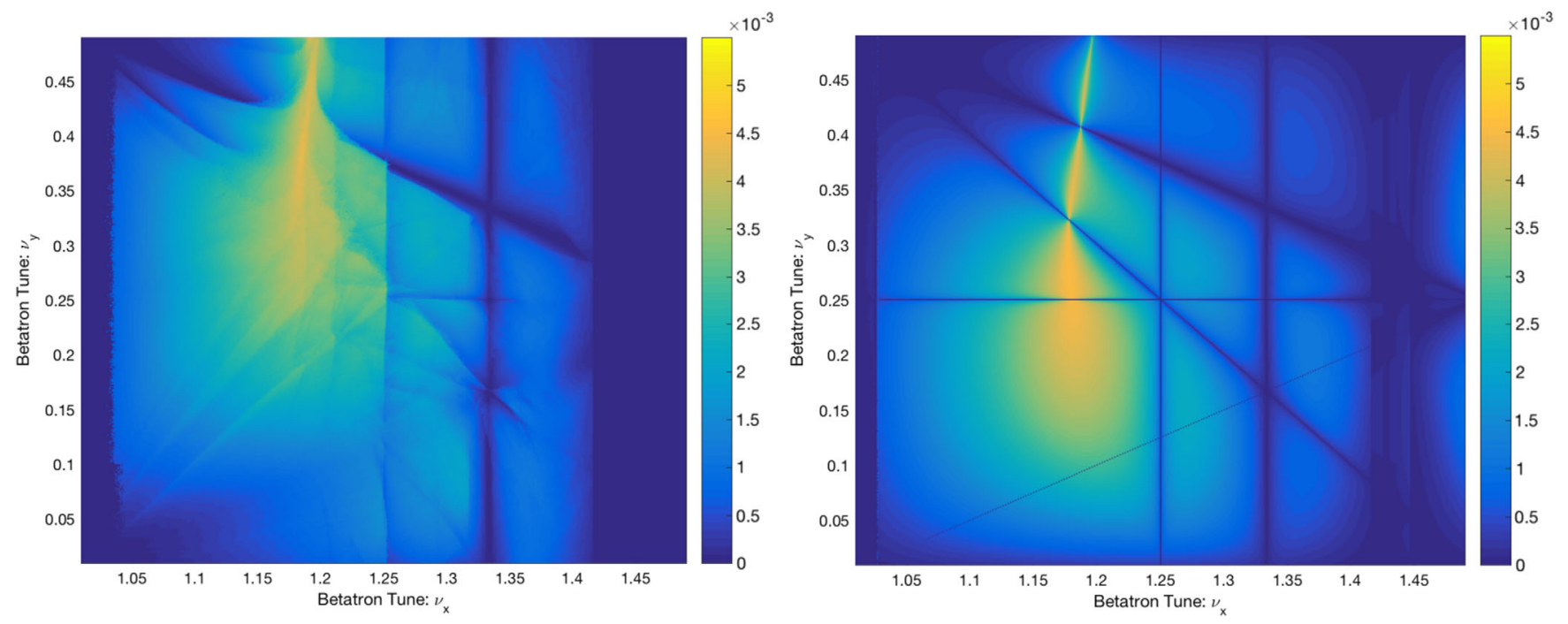

FIG. 10. Comparison of the dynamic aperture in the normalized coordinates in tracking (left) and a statistical model based on the third- and fourth-order resonances driving terms in the effective Hamiltonian (right).

where $C_{3}^{(r m s)}$ and $C_{4}^{(r m s)}$ are the rms values of the thirdand fourth-order driving terms in Eqs. (16) and (17), respectively. The function forms are obtained by the single-resonance analysis. Here we simply replace the single-resonance driving term with the rms value, assuming each term acts incoherently. Naturally, the smaller aperture dominates, and, therefore, the combined dynamic aperture can be written as

$$
a=\frac{2}{\frac{1}{a_{3}}+\frac{1}{a_{4}}} .
$$

The estimated dynamic aperture as a function of the betatron tunes is shown in the right plot in Fig. 10. In comparison to the tracking in the left plot, it clearly captures the most important features in the tune plane, especially the effects of the resonances. The smearing of the resonances seen in the tracking is presumably due to the tune shifts from Eq. (18) which are not in the formula.

The dynamic aperture of the cell with the parameters in Table I is between 6 and $7 \mathrm{~mm}$ in the horizontal plane, which is not quite adequate for off-axis injection as shown in Fig. 11.

\section{Optimization}

We introduce two families of harmonic sextupoles in the straight section: one positioned at the focusing quadrupoles and another at the defocusing quadrupoles. They are called $\mathrm{SF}$ and SD, respectively. We scan the dynamic aperture and compute the third-order driving terms while varying their strengths. The scanning results of the averaged dynamic aperture and $a_{3}$ are shown in the left and right plots in Fig. 12, respectively. As one can see from the figure, the agreement of the optimal settings of the harmonic sextupoles is excellent.
Since the third-order driving terms have linear dependences on the strengths of the sextupoles, we can formulate the optimization of $a_{3}$ according to a minimization of

$$
\chi^{2}=\sum_{i j}\left(C_{i}+A_{i j} S_{j}\right)\left(C_{i}+A_{i j} S_{j}\right)
$$

where $C_{i}$ represents the driving terms without the harmonic sextupoles, $S_{j}$ strengths of sextupoles, and $A_{i j}$ elements of the respond matrix. And then, the solution in matrix form is given by

$$
S=-\left(A^{T} A\right)^{-1}\left(A^{T} C\right),
$$

where the superscript " $T$ " notes the transpose of the matrix.

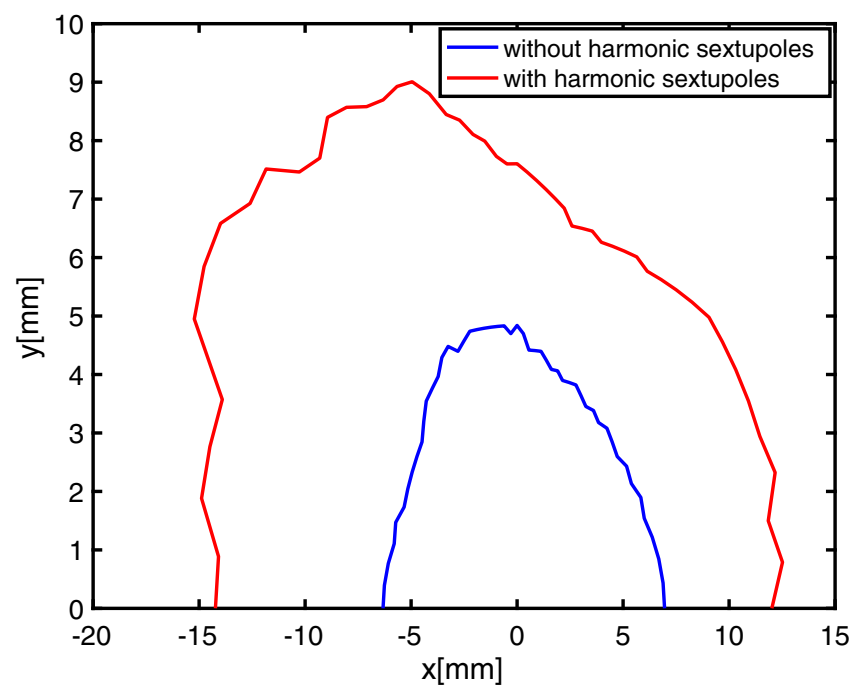

FIG. 11. A comparison of dynamic apertures with and without harmonic sextupoles. 

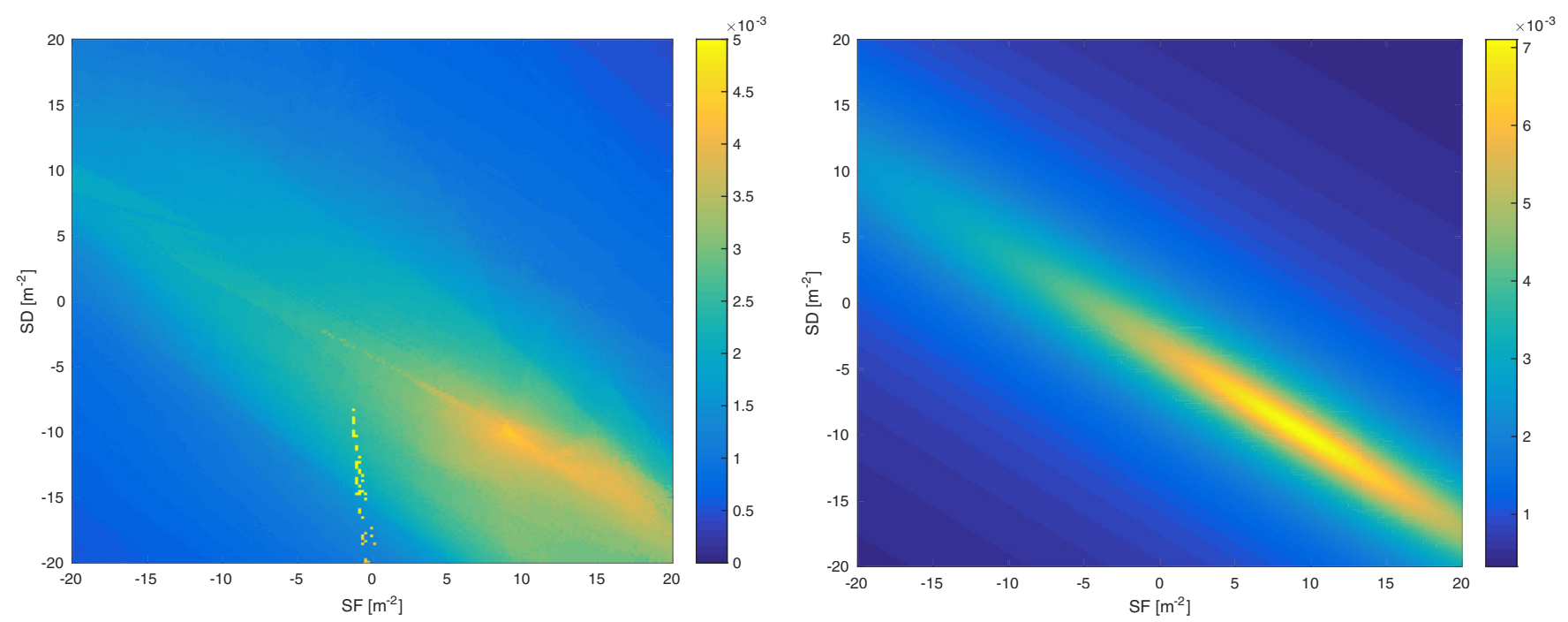

FIG. 12. Harmonic sextupole scanning of the dynamic aperture in the normalized coordinates in tracking (left) and the statistically estimated aperture $a_{3}$ based on the third-order resonance driving terms (right).
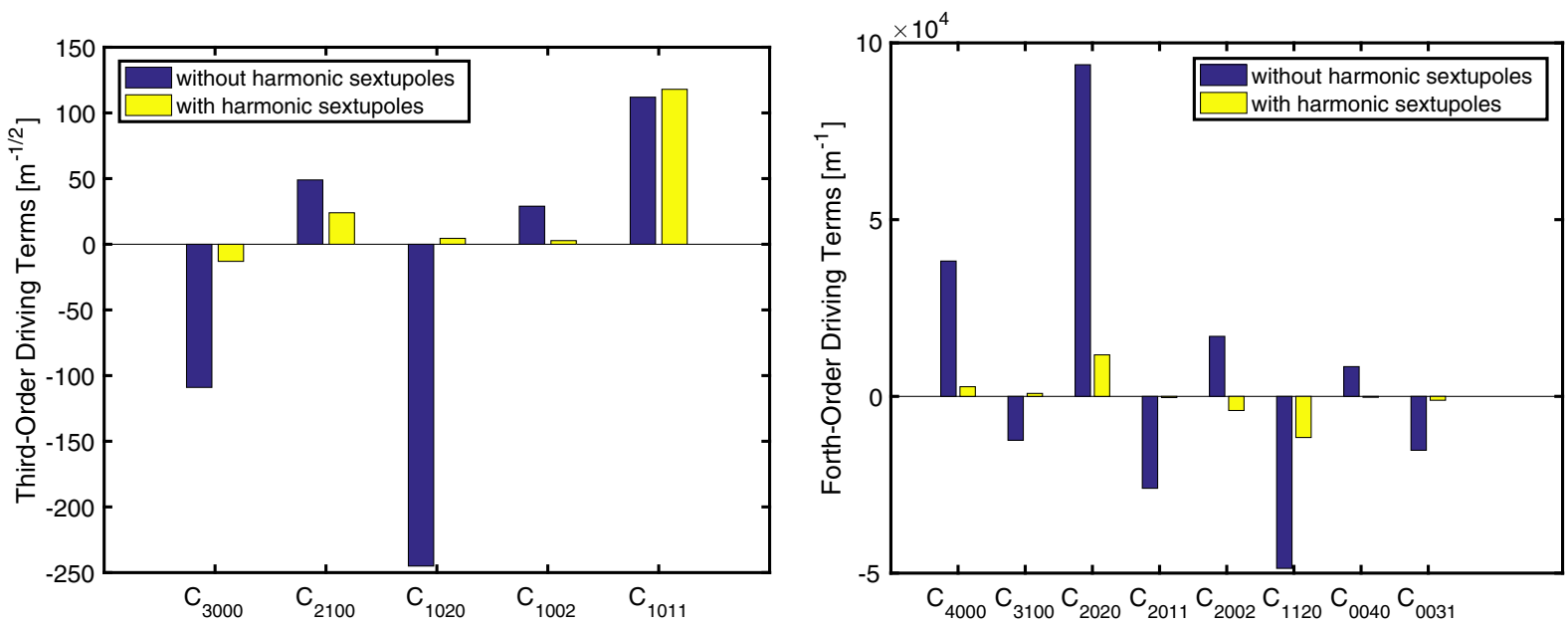

FIG. 13. A comparison of the third- and fourth-order resonance driving terms with and without harmonic sextupoles.
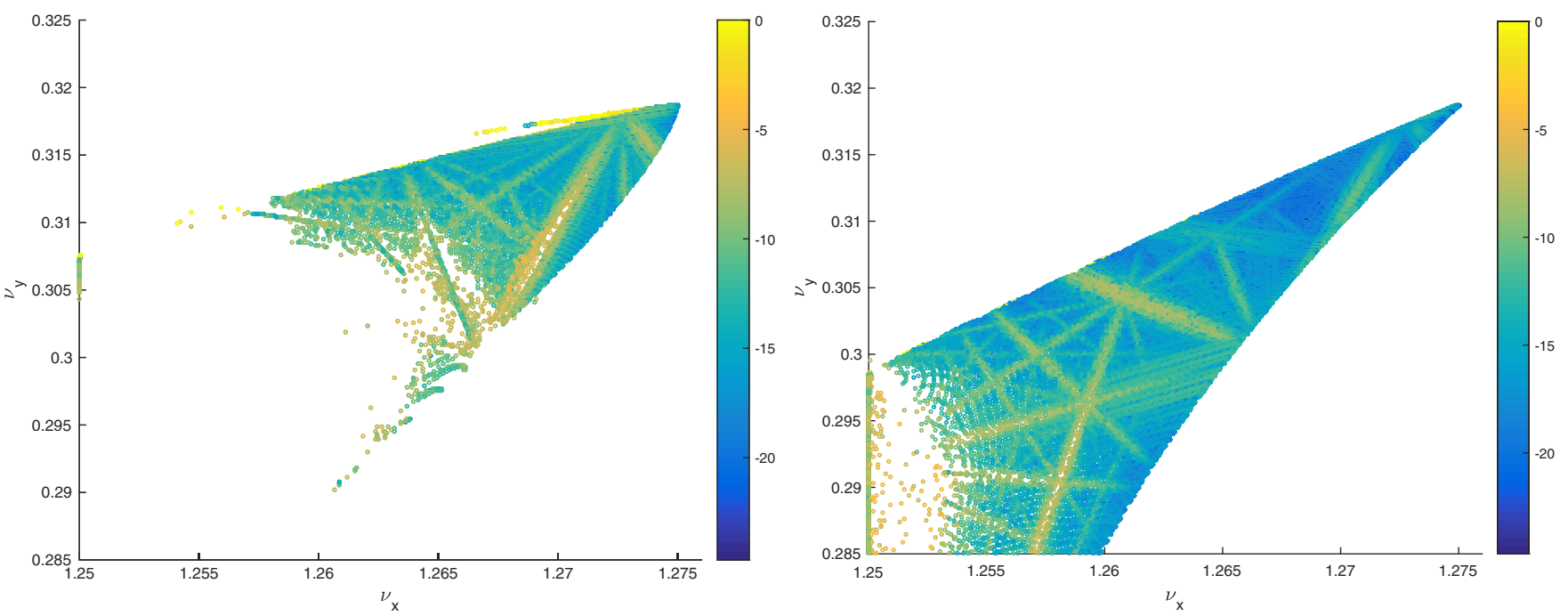

FIG. 14. Frequency map analysis of the beam footprints in the betatron tune plane without (left) and with (right) harmonic sextupoles. The color represents a diffusion rate in the logarithmic scale. 
We numerically compute them and find $\mathrm{SF}=8.903 \mathrm{~m}^{-2}$ and $\mathrm{SD}=-9.6396 \mathrm{~m}^{-2}$. As expected, they agree with the position of the peak seen in the right plot in Fig. 12. With these optimal settings of the harmonic sextupoles, the dynamic aperture increases dramatically as shown in Fig. 11. The improvement is much larger than the one seen in Ref. [17]. Moreover, we calculate the driving terms and show them in Fig. 13 for a comparison with and without the harmonic sextupoles. As a pleasant surprise, we see the significant reduction of not only the third-order terms but also the fourth-order ones. A similar improvement can be seen in the frequency map analysis [8] shown in Fig. 14. The diffusion rate $\log \sqrt{d \nu_{x}^{2}+d \nu_{y}^{2}}$ is computed from the tune changes between two consecutive 1020 turns. Such a large increase of the beam footprint far away from the corrected third-order resonances is another pleasant surprise, emphasizing the dominance of the low-order resonances.

\section{CONCLUSION}

We have comprehensively studied the simplest doublebend achromat cell with three families of quadrupoles. The cell is parametrized with the phase advances and the horizontal beta functions at the entry and center as well as the physical parameters in the dispersive region. Using the parametrization, we derive the minimum emittance.

With chromaticity set to zero by two families of sextupoles, we numerically computed the driving terms of the nonlinear resonance up to fourth order in the effective Hamiltonian, from which a simple formula of a dynamic aperture is constructed. The formula has captured the essential features in the two-dimensional tune scan by tracking.

Moreover, we have shown that an optimal dynamic aperture can be found simply by minimizing the driving terms of the third-order resonance with equal weight. The success shows the importance of including the information of how far away the resonance is from the operating point in the tune plane. Naturally, the effective Hamiltonian should be used to define the driving terms.

We have found all stable solutions for the simplest double-bend cell in terms of the roots of the cubic equations. The solution is not unique. Some branches have not been seen before, and they perhaps should be explored systematically later. More importantly, one can apply our results to understand the parameter space of the hybrid multibend achromat [34], which can be decomposed into a DBA and a " $\pi$ " cell. Naturally, our parametrization is suitable for the DBA and partially the $\pi$ cell because of the symmetrical feature of the dispersion bump where the sextupoles resides. Of course, one can also extend our results to a general DBA with more quadrupoles and further to the multibend achromat [35]. Given the complicity of our solutions, harder works are expected.

\section{ACKNOWLEDGMENTS}

I thank Yuri Nosochkov and Robert Warnock for helpful discussions. This work was supported by the Department of Energy under Contract No. DE-AC02-76SF00515.

\section{APPENDIX: SOLUTION OF THE CUBIC EQUATION}

Setting $x=L$, the stability condition in Eq. (12) can be rewritten as a cubic equation:

$$
a x^{3}+b x^{2}+c x+d=0,
$$

with the coefficients

$$
\begin{aligned}
a= & -8\left[(2 H-\mathrm{w}) \beta_{1} \cos \pi \nu-2 H \mathrm{w} \sin \pi \nu\right]\left[2 H \beta_{1} \cos \pi \nu-w(2 H+\mathrm{w}) \sin \pi \nu\right] \\
& \times\left[(H-\mathrm{w}) \beta_{1} \cos \pi \nu+\mathrm{w}\left(\sqrt{\beta_{1} \beta_{2}}-H \sin \pi \nu\right)\right]\left[(H-\mathrm{w}) \beta_{1} \cos \pi \nu+\mathrm{w}\left(2 \sqrt{\beta_{1} \beta_{2}}-H \sin \pi \nu\right)\right], \\
b= & 4\left[(2 H-\mathrm{w}) \beta_{1} \cos \pi \nu-2 H \mathrm{w} \sin \pi \nu\right]\left[(H-\mathrm{w}) \beta_{1} \cos \pi \nu+\mathrm{w}\left(\sqrt{\beta_{1} \beta_{2}}-H \sin \pi \nu\right)\right] \\
& \times\left\{-7 H \mathrm{w}^{2} \beta_{1} \beta_{2}-7 \mathrm{w}^{3} \beta_{1} \beta_{2}+4 \mathrm{w} \sqrt{\beta_{1} \beta_{2}}\left[-H^{2} \beta_{1}+2 H \mathrm{w} \beta_{2}+\mathrm{w}^{2}\left(\beta_{1}+\beta_{2}\right)\right] \cos \pi \nu\right. \\
& -\mathrm{w}\left(-4 H^{2}+H \mathrm{w}+\mathrm{w}^{2}\right) \beta_{1} \beta_{2} \cos 2 \pi \nu+4 H \mathrm{w} \sqrt{\beta_{1} \beta_{2}}\left(H \mathrm{w}+\mathrm{w}^{2}+2 \beta_{1} \beta_{2}\right) \sin \pi \nu \\
& \left.-2 H^{2} \mathrm{w}^{2} \beta_{2} \sin 2 \pi \nu-H \mathrm{w}^{3} \beta_{2} \sin 2 \pi \nu+2 H^{2} \beta_{1}^{2} \beta_{2} \sin 2 \pi \nu-2 H \mathrm{w} \beta_{1}^{2} \beta_{2} \sin 2 \pi \nu\right\} \\
& +2\left[2 H \beta_{1} \cos \pi \nu-\mathrm{w}(2 H+\mathrm{w}) \sin \pi \nu\right]\left[(H-\mathrm{w}) \beta_{1} \cos \pi \nu+\mathrm{w}\left(2 \sqrt{\beta_{1} \beta_{2}}-H \sin \pi \nu\right)\right] \\
& \times\left\{-7 H \mathrm{w}^{2} \beta_{1} \beta_{2}-8 H \mathrm{w} \sqrt{\beta_{1} \beta_{2}}\left[H \beta_{1}-\mathrm{w}\left(\beta_{1}+\beta_{2}\right)\right] \cos \pi \nu+3 H(4 H-3 \mathrm{w}) \mathrm{w} \beta_{1} \beta_{2} \cos 2 \pi \nu\right. \\
& +8 H^{2} \mathrm{w}^{2} \sqrt{\beta_{1} \beta_{2}} \sin \pi \nu+8 H \mathrm{w}\left(\beta_{1} \beta_{2}\right)^{3 / 2} \sin \pi \nu-4 \mathrm{w}^{2}\left(\beta_{1} \beta_{2}\right)^{3 / 2} \sin \pi \nu \\
& \left.-6 H^{2} \mathrm{w}^{2} \beta_{2} \sin 2 \pi \nu+6 H^{2} \beta_{1}^{2} \beta_{2} \sin 2 \pi \nu-9 H \mathrm{w} \beta_{1}^{2} \beta_{2} \sin 2 \pi \nu+3 \mathrm{w}^{2} \beta_{1}^{2} \beta_{2} \sin 2 \pi \nu\right\} \\
& -4 \mathrm{w}^{3} \beta_{1} \beta_{2}\left[(H-\mathrm{w}) \beta_{1} \cos \pi \nu-H \mathrm{w} \sin \pi \nu\right]^{2} \sin ^{2} \pi \nu_{y},
\end{aligned}
$$




$$
\begin{aligned}
& c=-4 \beta_{2}\left[2 H \beta_{1} \cos \pi \nu-\mathrm{w}(2 H+\mathrm{w}) \sin \pi \nu\right]\left[H \mathrm{w} \cos \pi \nu+(H-\mathrm{w}) \beta_{1} \sin \pi \nu\right] \\
& \times\left[-2 H \mathrm{w} \sqrt{\beta_{1} \beta_{2}}+2 H \mathrm{w} \beta_{2} \cos \pi \nu+(2 H-\mathrm{w}) \beta_{1} \beta_{2} \sin \pi \nu\right] \\
& \times\left[(H-\mathrm{w}) \beta_{1} \cos \pi \nu+\mathrm{w}\left(2 \sqrt{\beta_{1} \beta_{2}}-H \sin \pi \nu\right)\right] \\
& +\left\{7 H \mathrm{w}^{2} \beta_{1} \beta_{2}+7 \mathrm{w}^{3} \beta_{1} \beta_{2}-4 \mathrm{w} \sqrt{\beta_{1} \beta_{2}}\left[-H^{2} \beta_{1}+2 H \mathrm{w} \beta_{2}+\mathrm{w}^{2}\left(\beta_{1}+\beta_{2}\right)\right] \cos \pi \nu\right. \\
& +\mathrm{w}\left(-4 H^{2}+H \mathrm{w}+\mathrm{w}^{2}\right) \beta_{1} \beta_{2} \cos \pi \nu-4 H \mathrm{w} \sqrt{\beta_{1} \beta_{2}}\left(H \mathrm{w}+\mathrm{w}^{2}+2 \beta_{1} \beta_{2}\right) \sin \pi \nu \\
& \left.+2 H^{2} \mathrm{w}^{2} \beta_{2} \sin 2 \pi \nu+H \mathrm{w}^{3} \beta_{2} \sin 2 \pi \nu-2 H^{2} \beta_{1}^{2} \beta_{2} \sin 2 \pi \nu+2 H \mathrm{w} \beta_{1}^{2} \beta_{2} \sin 2 \pi \nu\right\} \\
& \times\left\{-7 H \mathrm{w}^{2} \beta_{1} \beta_{2}-8 H \mathrm{w} \sqrt{\beta_{1} \beta_{2}}\left[H \beta_{1}-\mathrm{w}\left(\beta_{1}+\beta_{2}\right)\right] \cos \pi \nu+3 H(4 H-3 \mathrm{w}) \mathrm{w} \beta_{1} \beta_{2} \cos 2 \pi \nu\right. \\
& +8 H^{2} \mathrm{w}^{2} \sqrt{\beta_{1} \beta_{2}} \sin \pi \nu+8 H \mathrm{w}\left(\beta_{1} \beta_{2}\right)^{3 / 2} \sin \pi \nu-4 \mathrm{w}^{2}\left(\beta_{1} \beta_{2}\right)^{3 / 2} \sin \pi \nu \\
& \left.-6 H^{2} \mathrm{w}^{2} \beta_{2} \sin 2 \pi \nu+6 H^{2} \beta_{1}^{2} \beta_{2} \sin 2 \pi \nu-9 H \mathrm{w} \beta_{1}^{2} \beta_{2} \sin 2 \pi \nu+3 \mathrm{w}^{2} \beta_{1}^{2} \beta_{2} \sin 2 \pi \nu\right\} \\
& +8 \mathrm{w}^{3} \beta_{1} \beta_{2}^{2}\left[-(H-\mathrm{w}) \beta_{1} \cos \pi \nu+H \mathrm{w} \sin \pi \nu\right]\left[-H \mathrm{w} \cos \pi \nu+(-H+\mathrm{w}) \beta_{1} \sin \pi \nu\right] \sin ^{2} \pi \nu_{y} \text {, } \\
& d=2 \beta_{2}\left[H \mathrm{w} \cos \pi \nu+(H-\mathrm{w}) \beta_{1} \sin \pi \nu\right] \\
& \times\left\{\left[2 H \mathrm{w} \sqrt{\beta_{1} \beta_{2}}-2 H \mathrm{w} \beta_{2} \cos \pi \nu+(-2 H+\mathrm{w}) \sin \pi \nu\right]\right. \\
& \times\left[7 H \mathrm{w}^{2} \beta_{1} \beta_{2}+7 \mathrm{w}^{3} \beta_{1} \beta_{2}-4 \mathrm{w} \sqrt{\beta_{1} \beta_{2}}\left(-H^{2} \beta_{1}+2 H \mathrm{w} \beta_{2}+\mathrm{w}^{2} \beta_{1}+\mathrm{w}^{2} \beta_{2}\right) \cos \pi \nu\right. \\
& +\mathrm{w}\left(-4 H^{2}+H \mathrm{w}+\mathrm{w}^{2}\right) \beta_{1} \beta_{2} \cos 2 \pi \nu-4 H \mathrm{w} \sqrt{\beta_{1} \beta_{2}}\left(H \mathrm{w}+\mathrm{w}^{2}+2 \beta_{1} \beta_{2}\right) \sin \pi \nu \\
& \left.+2 H^{2} \mathrm{w}^{2} \beta_{2} \sin 2 \pi \nu+H \mathrm{w}^{3} \beta_{2} \sin 2 \pi \nu-2 H^{2} \beta_{1}^{2} \beta_{2} \sin 2 \pi \nu+2 H \mathrm{w} \beta_{1}^{2} \beta_{2} \sin 2 \pi \nu\right] \\
& \left.-2 \mathrm{w}^{3} \beta_{1} \beta_{2}^{2}\left[H \mathrm{w} \cos \pi \nu+(H-\mathrm{w}) \beta_{1} \sin \pi \nu\right] \sin ^{2} \pi \nu_{y}\right\} \text {. }
\end{aligned}
$$

The solution of the cubic equation is well known. One needs to compute first

$$
\begin{aligned}
& \Delta_{0}=b^{2}-3 a c, \\
& \Delta_{1}=2 b^{2}-9 a b c+27 a^{2} d, \\
& \Omega=\frac{\Delta_{1} \pm \sqrt{\Delta_{1}^{2}-4 \Delta_{0}^{3}}}{2} .
\end{aligned}
$$

Then the roots are given by

$x_{k}=-\frac{1}{3 a}\left(b+\xi^{k} \Omega^{1 / 3}+\frac{\Delta_{0}}{\xi^{k} \Omega^{1 / 3}}\right), \quad k \in\{0,1,2\}$,

where $\xi=-\frac{1}{2}+\frac{i}{2} \sqrt{3}$.

[1] R. Chasman, G. K. Green, and E. M. Rowe, Preliminary design of dedicated synchrotron radiation facility, IEEE Trans. Nucl. Sci. 22, 1765 (1975).

[2] D. Einfeld and G. Mulhaupt, Choice of the principal parameters and lattice of BESSY, Nucl. Instrum. Methods Phys. Res., Sect. A 172, 55 (1980).

[3] E. Rowe, M. A. Green, J. W. Hicks, W. S. Trzeciak, and W. R. Winter, Status of the Aladdin project, IEEE Trans. Nucl. Sci. 28, 3145 (1981).
[4] M. Sommer, Optimizing of the emittance of electrons (positrons) storage rings, Report No. LAL/RT/83-15, LAL, 1983.

[5] J. Murphy, Synchrotron Light Source Data Book, BNL 42333, version 4.0 (Brookhaven National Laboratory, Brookhaven, 1996).

[6] H. Grote and F. C. Iselin, The MAD program (methodical accelerator design) version 8.15, Report No. CERN/SL/ 90-13 (AP), 1990.

[7] D. S. Robin, W. Wan, F. Sannibale, and V. P. Suller, Global analysis of all linear settings of a storage ring lattice, Phys. Rev. Accel. Beams 11, 024002 (2008).

[8] J. Laskar, The chaotic motion of the Solar System: A numerical estimate of the size of the chaotic zones, Icarus 88, 266 (1990).

[9] D. Robin, C. Steier, J. Laskar, and L. Nadolski, Global Dynamics of Advanced Light Source Revealed through Experimental Frequency Analysis, Phys. Rev. Lett. 85, 558 (2000).

[10] L. Nadolski and J. Laskar, Review of single particle dynamics for third generation light sources through frequency map analysis, Phys. Rev. Accel. Beams 6, 114801 (2003).

[11] L. Tosi et al., Optics and transverse beam dynamics in ELETTRA, in Proceedings of the Fourth European Particle Accelerator Conference EPAC 94, London, England (World Scientific, River Edge, NJ, 1994).

[12] J. Bengtsson, The sextupole scheme for the Swiss Light Source (SLS): An analytical approach, SLS Note No. 9/97, 1997.

[13] L. Farvacque, T. F. Gunzel, J. L. Laclare, and A. Ropert, BETA Users' Guide, 3rd ed. (CEA-Saclay, France, 2001). 
[14] A. Streun, OPA lattice design code, PSI, Switzerland, 2012.

[15] J. Ablett et al., NSLS-II conceptual design report, technical report, Brookhaven, 2006, https://www.bnl.gov/nsls2/ project/CDR.

[16] S. C. Leemann, Å. Andersson, M. Eriksson, L.-J. Lindgren, E. Wallén, J. Bengtsson, and A. Streun, Beam dynamics and expected performance of Sweden's new storage-ring source: MAX IV, Phys. Rev. Accel. Beams 12, 120701 (2009).

[17] A. Sharma, D. K. Tyagi, and A. D. Ghodke, Optimization of harmonic sextupoles in Indus-2 electron storage ring, Nucl. Instrum. Methods Phys. Res., Sect. A 782, 28 (2015).

[18] M. Borland et al., Multi-objective direct optimization of dynamic acceptance and lifetime for potential upgrades of the Advanced Photon Light Source, APS Report No. 319, Argonne, 2010.

[19] L. Yang, Y. Li, W. Guo, and S. Krinsky, Multiobjective optimization of dynamic aperture, Phys. Rev. Accel. Beams 14, 054001 (2011).

[20] Y. Li, W. Cheng, L. H. Yu, and R. Rainer, Genetic algorithm enhanced by machine learning in dynamic aperture optimization, Phys. Rev. Accel. Beams 21, 054601 (2018).

[21] Y. Cai, Singularity and stability in a periodic system of particle accelerators, Phys. Rev. Accel. Beams 21, 054002 (2018).

[22] Y. Cai, Single-particle dynamics in theoretical minimum emittance cell, Phys. Rev. Accel. Beams 21, 114002 (2018).

[23] D. A. Edwards and M. J. Syphers, An Introduction the Physics of High Energy Accelerators (Wiley, New York, 1993).

[24] E. D. Courant and H. S. Snyder, Theory of the alternatinggradient synchrotron, Ann. Phys. (Paris) 3, 1 (1958).
[25] Y. Cai, Symplectic maps and chromatic optics in particle accelerators, Nucl. Instrum. Methods Phys. Res., Sect. A 797, 172 (2015).

[26] H. Wiedemann, Radiation integrals, in Handbook of Accelerator Physics and Engineering, 2nd ed., edited by A. Chao, K. H. Mess, M. Tigner, and F. Zimmermann (World Scientific, Singapore, 2013), p. 220.

[27] A. A. Fakhri, P. Kant, G. Singh, and A. D. Ghodke, An analytical study of double bend achromat lattice, Rev. Sci. Instrum. 86, 033304 (2015).

[28] A. Chao, Lecture notes on topics in accelerator physics, Report No. SLAC-PUB-9574, 2002.

[29] A. J. Dragt, Lie algebraic theory of geometrical optics and optical aberrations, J. Opt. Soc. Am. 72, 372 (1982); A. Dragt, F. Neri, G. Rangarajan, D. R. Douglas, L. M. Healy, and R. D. Ryne, Lie algebraic treatment of linear and nonlinear beam dynamics, Annu. Rev. Nucl. Part. Sci. 38, 455 (1988).

[30] A. J. Dragt and J. M. Finn, Normal form for mirror machine Hamiltonian, J. Math. Phys. (N.Y.) 20, 2649 (1979).

[31] E. Forest, M. Berz, and J. Irwin, Normal form methods for complicated periodic systems: A complete solution using differential algebra and Lie operators, Part. Accel. 24, 91 (1989).

[32] R. D. Ruth, Single-particle dynamics in circular accelerator, AIP Conf. Proc. 153, 150 (1985).

[33] M. Berz, Differential algebra description of beam dynamics to very high order, Part. Accel. 24, 109 (1989).

[34] P. Raimondi, Hybrid multi bend achromat: From SuperB to EBC, in Proceedings of IPAC2017, Copenhagen, Denmark (JACoW, 2017).

[35] D. Einfeld, M. Plesko, and J. Schaper, First multi-bend achromat lattice consideration, J. Synchrotron Radiat. 21, 856 (2014). 